\title{
Iconografía musical en el camarín de Santa Ana de Sapa (Manila)
}

\section{Musical iconography in the chapel of Santa Ana de Sapa (Manila)}

\author{
Pedro LUENGO GUTIÉRREZ \\ Proyecto I+D “Iconografía musical” UCM. Becario FPU. Universidad de Sevilla \\ pedroluengo@us.es
}

Recibido: 02/06/2012

Aprobado: 28/07/2012

Resumen: La escasez de representaciones artísticas conservadas en Filipinas dificulta el estudio de la iconografía musical y, con ello, de las prácticas interpretativas y constructivas de los instrumentos. El análisis de este conjunto pictórico permite ofrecer nuevos datos sobre la producción pictórica y la representación musical en los territorios asiáticos del mundo hispánico.

Palabras clave: Filipinas, ángeles músicos, arpa, guitarra-lira.

Abstract: The shortage of artistic images preserved in the Philippines renders difficult the study of musical iconography and thus the practices of interpretation and construction of the instruments. The analysis of this pictorial set allows to offer new insights into the pictorial and musical production in the Asian territories of the Hispanic world.

Kewy Words: Philippines, musical angels, harp, guitar-lyre

En los últimos años se han multiplicado en España los estudios sobre iconografía musical, afrontando los estilos y zonas más distantes, y mostrando a su vez un vasto campo de investigación. Un capítulo de gran importancia en el estudio de la representación musical hispánica es el conocimiento de lo conservado en territorios americanos y asiáticos que estuvieron vinculados a la monarquía hispana ${ }^{1}$. En tal sentido, las recientes publicaciones sobre estos temas en México serán fundamentales no sólo para la comprensión de los modelos novohispanos, sino particularmente para la de los filipinos. Al contrario que en el país mesoamericano, el estudio de la iconografía musical en Filipinas apenas está iniciado, por lo que no se conocen publicaciones monográficas al respecto. Hasta cierto punto esta realidad es sencilla de explicar historiográficamente. De las creaciones artísticas más desarrolladas en el archipiélago sin duda han sido los marfiles los que mayor atención han recibido, sin que en este tipo de piezas se hayan encontrado representaciones de instrumentos musicales, a pesar del elevado número de ellas ${ }^{2}$. La escultura en otros materiales, aprovechándose de la variedad y calidad de maderas fácilmente extraíbles en las islas, sí se desarrolló en algunas zonas, tanto en retablos como en imágenes de candelero. La pintura, quizás junto a la escultura, la más propicia para la iconografía musical, se conserva con enorme

\footnotetext{
${ }^{1}$ Para el caso mexicano se pueden destacar distintos estudios. como Álvarez, Rosario. "La iconografía musical latinoamericana en el renacimiento y el barroco: importancia y pautas para su estudio". Interamer, $\mathrm{n}^{\circ}$ 26. Washington, 1993. Reyes, Octavio. Iconografía musical novohispana en la región central de Puebla. Puebla: Universidad Iberoamericana, Plantel Golfo-Centro, 1995; Roubina, Eugenia. "Fuentes iconográficas en el estudio de las prácticas instrumentales en la música popular novohispana del siglo XVIII". Cátedra de Artes, n 4. Santiago de Chile: Pontificia Universidad Católica de Chile, 2007.

${ }^{2}$ Para el estudio de los marfiles filipinos debe consultarse Estella Marcos, Margarita. La escultura barroca de marfil en España: las escuelas europeas y las coloniales. Madrid: CSIC, 1984; entre otras obras de esta investigadora.
} 
dificultad en el archipiélago, siendo excepcionales las piezas anteriores al siglo XVIII, y escasísimas las de esta centuria ${ }^{3}$.

Una vez vistas someramente las posibilidades artísticas que ofrecía el archipiélago, es necesario prestar atención a la realidad musical filipina durante los siglos XVII y $\mathrm{XVIII}^{4}$. Como en otros territorios hispanoamericanos, las celebraciones religiosas, y por tanto sus coros y capillas musicales, fueron fundamentales en el devenir musical de la sociedad del archipiélago. La actividad organística queda demostrada por los instrumentos conservados y/o conocidos, mientras que la música vocal, plasmada en distintos libros de coro, ha sido estudiada recientemente ${ }^{5}$. Por otra parte, aunque aún poco conocida, hay que subrayar la música profana. Es posible que las bandas musicales de los regimientos militares tuvieran un papel acorde con la relevancia de la milicia en las islas. Además, queda patente en distintos cronistas la afición y facilidad de los nativos hacia la música ${ }^{6}$. Las fiestas, entre las que hay que incluir celebraciones paralitúrgicas, como las procesiones o los recibimientos de personalidades, fueron amenizadas durante el siglo XIX con agrupaciones "de distintas naciones", lo que evidencian la presencia de instrumentos chinos en el archipiélago ${ }^{7}$.

Para el estudio de la práctica musical en los conventos de Filipinas pueden usarse, entre otras fuentes, documentos de regulación interna de las distintas órdenes religiosas. Teniendo en cuenta que, como se verá más adelante, Santa Ana de Sapa es desde su fundación un convento franciscano, y que esta orden tenía estatutos referentes al tema musical, es necesario tratarlos. Según los publicados en 1753, redactados en el Definitorio de 1730, no se permitía tener órganos en los conventos de comunidad ${ }^{8}$. Solo se podían usar en las doctrinas o ministerios, previa licencia del prelado provincial. Esta prohibición, que, como se verá, en los mismos estatutos no debió tener total implantación, se ampliaba a otros instrumentos con los que los indios cantores "ofician la misa y demás divinas alabanzas". De entre este grupo de instrumentos, que, al parecer, debía ser bastante amplio, los estatutos citan el arpa, que en momento tan temprano parece tener ya un gran desarrollo en las islas. En el capítulo V de los mismos estatutos, se permite tocar el órgano mientras se dice la misa, así como otros instrumentos según costumbre del lugar, con lo cual, se consiente la música dentro de

\footnotetext{
${ }^{3}$ Sobre las distintas manifestaciones artísticas filipinas hay que destacar las siguientes publicaciones. Javellana, René B.: Wood \& Stone for God's greater glory. Jesuit art \& architecture in the Philippines. Quezon City: Ateneo de Manila University Press, 1991; Trota José, Regalado: Simbahn: Church art in colonial Philippines. 1565-1898. Manila: Ayala Foundation, 1992. VV.AA.: Manila 1571-1898. Occidente en Oriente. Madrid: Ministerio de Fomento, 1998; AA.VV. Igkas Arte. The Philippine Arts during the Spanish Period. Manila: CCP-AECI, 1998. Martínez Shaw, Carlos y Alfonso Mola, Marina (dir.): Catálogo de la exposición El Galeón de Manila. Sevilla: MECD y Focus-Abengoa, 2000. Galende, Pedro G. y Trota José, Regalado: San Agustín. Art \& History. 1571-2000”. Hong Kong, 2000. Morales, Alfredo J. (dir.): Catálogo de la exposición Filipinas, puerta de Oriente. De Legazpi a Malaspina. Madrid: SEACEX, 2003. Ruiz Gutiérrez, Ana. El tráfico artístico entre España y Filipinas (1565-1815). Granada: Universidad de Granada, 2005.

${ }^{4}$ Irving, David: Colonial Counterpoint: Music in Early Modern Manila. Nueva York-Oxford: Oxford University Press, 2010; "Some Notes on Viols in the Philippines". Chelys Australis: Australian Viola da Gamba Society Journal, 4 (2004). Pp. 13-16; "Keyboard instruments and instrumentalists in Manila, 1581-1798”. Anuario Musical, 60. Madrid, 2005. Pp. 27-40.

${ }^{5}$ Irving, David: Colonial Counterpoint...

${ }^{6}$ Quizás el caso más llamativo para el presente estudio es la formación musical que recibieron del mártir Fr. Pedro Bautista a finales del siglo XVI los nativos de Namayan, futuro Santa Ana de Sapa, relatado por Huerta, Félix de. Estado geográfico, topográfico, estadístico, histórico-religioso... Manila: Sánchez y Cía., 1865.

${ }^{7}$ Moreno Garbayo, Justa. Fiestas en Manila año 1825. Madrid: Patrimonio nacional, 1977.

${ }^{8}$ Estatutos y ordenaciones de la Santa Provincia de San Gregorio... Sampaloc: Convento de Nuestra Señora de Loreto, 1753. Cap. IV
} 
los conventos franciscanos con bastante libertad en lo que a instrumentación se refiere ${ }^{9}$. Como ya se comentará, el arpa cobró un desarrollo importante en la zona ilocana hasta la provincia de Cebú, incluyendo evidentemente la capital. Distintas obras, como la que se representa en Santa Ana de Sapa, parecen demostrar que la tipología se seguía con precisión también en Manila. Todos estos elementos y otros que se irán señalando serán fundamentales para la comprensión de la producción iconográfica a estudiar.

Para el estudio de la iconografía musical en Filipinas se ha considerado pertinente elegir una pieza representativa conservada en Manila. Al respecto resulta interesante la decoración pictórica del techo del camarín de la Virgen de los Desamparados, tras el presbiterio del convento franciscano de Santa Ana de Sapa (Manila). El convento fue fundado por los propios franciscanos en 1578, aunque no sería hasta mucho más tarde cuando empezó a construirse en piedra ${ }^{10}$. De todas formas, según puede desprenderse de nueva documentación localizada, la fábrica del convento se benefició de un importante impulso económico en el siglo XVII. En el testamento de Juan Pardo de Losada y Quiroga, fechado en 1625, se encuentra una donación para la fundación de un convento e iglesia en Santa Ana de Sapa (Manila) ${ }^{11}$. Este cenobio franciscano serviría, según el mismo documento, como enterramiento de este general gallego. Ya en el siglo XVIII, el convento se convertiría en uno de los centros más importantes de la orden en la zona, tras la propia casa provincial en Intramuros. La dirección de esta obra está atribuida tradicionalmente a fray Vicente Inglés, y la primera piedra del edificio se puso el 12 de septiembre de $1720^{12}$. Es probable que este provincial franciscano sólo fuera el alma mater del proyecto de reconstrucción de la iglesia y convento, pero es poco factible que las tareas propias de su cargo en una provincia como la de San Gregorio de Filipinas le permitieran dirigir un proyecto de tal calibre ${ }^{13}$. El costo total ascendió a 33.000 pesos, que se cubrirían con los 4.000 aportados por el gobernador general, los aportes del propio provincial franciscano y donaciones en general. En el presbiterio del recién estrenado convento se enterraron el obispo franciscano Pedro de la Santísima Trinidad Martínez y Arizala, en 1755, y el citado Vicente Inglés, en 1759. Probablemente la iglesia sea más conocida por las famosas excavaciones en ella desarrolladas y de las que se recuperó una importante colección de piezas de porcelana china, o por el magnífico retablo, probablemente realizado durante el proceso de construcción de la iglesia en el siglo XVIII, que por el imponente camarín del que trata este trabajo. Ya en el siglo XX, entre 1977 y 1985, los arquitectos Juan Napkil, Jaime Pilar y el ingeniero Arturo Mañalac dirigieron las obras de renovación del edificio.

La reconstrucción de Santa Ana, comenzada en los primeros años del siglo XVIII, forma parte del proceso de renovación arquitectónica desarrollada en Manila tras muchas décadas de escasa actividad constructiva. De hecho, la misma orden seráfica estaba construyendo en estos momentos la casa provincial, el otro convento de la ciudad, el de San Francisco, en Intramuros. Este aumento en el número y calidad de los proyectos se debe al descenso de los desastres naturales -baguíos (huracanes) y terremotos fundamentalmente-, y a una mejora de la situación económica y comercial

\footnotetext{
${ }^{9}$ Estatutos y ordenaciones de la Santa Provincia de San Gregorio... Sampaloc: Convento de Nuestra Señora de Loreto, 1753. Cap. V

${ }^{10}$ AA.VV. Santa Ana Church. A Historical Guide. Manila: Cofradía de la Inmaculada Concepción Foundation, 2008.

${ }^{11}$ AGI, CONTRATACION, 524, N.2, R.10.

${ }_{12}^{12}$ Galende, Pedro G.: Philippine Church Façades. Manila, San Agustín Museum, 2008, p. 15.

13 Además, en caso de tener conocimientos técnicos de cierta relevancia, habría participado activamente en el proceso de construcción de otras iglesias franciscanas de igual o mayor calibre cercanas geográficamente.
} 
del archipiélago. El camarín se encuentra en el segundo piso del retablo mayor y actualmente resguarda a Nuestra Señora de los Desamparados, devoción de origen valenciano, de donde por otra parte procedía el propio Inglés. Según parece, la imagen se realizaría en España en 1713 y llegaría a Filipinas en 1725 traída por Vicente Inglés ${ }^{14}$. Como es tradicional en este tipo de esculturas devocionales, las manos y el rostrillo son de madera, mientras que el resto de la pieza es un candelero. En lo que a arquitectura se refiere es un espacio de gran interés, cuyo estudio rebasa los límites de este trabajo. El esquema arquitectónico parece estar basado en el de camarín-torre, lo que sería un nuevo ejemplo dentro de la arquitectura filipina, junto al coetáneo y recientemente documentado de Tondo en $1727^{15}$. De todas formas, resulta evidente que ha sufrido un buen número de reformas a lo largo de su historia, seguramente debido al desarrollo de la propia devoción. Es posible que la virgen que llegara de España comenzara siendo el remate de una obra de gran nivel como era Santa Ana. Con el paso de la segunda mitad del siglo XVIII, la devoción crecería entre la feligresía, lo que conllevaría la modificación del retablo mayor y la reforma del camarín y sus respectivos accesos, para lo que es probable que se reutilizaran construcciones anteriores.

Las pinturas del camarín son anónimas y aún no ha podido documentarse su fecha de realización. Tras un análisis formal y algunas comparaciones con otras piezas, parece claro que las pinturas no pueden datarse en las mismas fechas que la construcción de la iglesia, ni siquiera en lo que podría considerarse una segunda etapa en la realización del exorno escultórico de la misma. Deben por tanto considerarse como obras decimonónicas contemporáneas de Damián Domingo, o un poco anteriores aunque en ningún caso atribuibles a su mano ${ }^{16}$. Estilísticamente dejan claro su relación con obras de principios del siglo XIX, como es el caso del pintor citado, aunque la propia iconografía musical hace adelantar ligeramente esta posibilidad. Instrumentos como la chirimía o la corneta, que además aparecen en gran número, deben adscribirse a prácticas musicales dieciochescas dentro del archipiélago. Por otra parte, instrumentos como la guitarra o los vientos metales en general, que pueden observarse en otras piezas filipinas datadas en las primeras décadas del siglo XIX, no aparecen en Santa Ana ${ }^{17}$.

\footnotetext{
${ }^{14}$ Bajo la base de la Virgen se encuentra una nota con la siguiente inscripción "Copia verdadera de la imagen milagrosa de Maria, ayuda de los desamparados, hecha en la Ciudad de Valencia, España, en 1713 y bendecida por ser tocada con la imagen original realizada por tres ángeles en 1416", lo que la relaciona evidentemente con la devoción levantina más comúnmente conocida como "la Geperudeta".

${ }^{15}$ El de Santa Ana sería en cualquier caso posterior a la llegada de la imagen en 1725 , por lo que puede considerarse que la estructura original fuera contemporánea a la de Tondo. Luengo, Pedro. "Noticias sobre obras en la iglesia de Tondo en el siglo XVIII". Laboratorio de Arte. Sevilla, 2010. -en prensa-. Kubler estableció una clasificación en la que distinguió entre el tipo oculto y el camarín-torre, habiéndose aceptado el término según se constata en publicaciones posteriores. Kubler, George. Arquitectura de los siglos XVII y XVIII, Ars Hispaniae XIV, Madrid, 1957; Id., "El camarín del Siglo de Oro" en Cuadernos Hispanoamericanos, no 140-141. 1961, pp. 239-245.

${ }^{16}$ La labor de Damián Domingo como pintor religioso es poco conocida debido al escaso número de obras de esta temática dentro de su catálogo (Cátedra de San Pedro, Virgen del Rosario, Apoteosis de Santo Tomás y Sagrada Familia). La Sagrada Familia demuestra que su forma de componer y su dibujo distan mucho del de este camarín. Por otra parte la tabla de la Apoteosis, obra de mayor tamaño del pintor filipino, comparte con Santa Ana el grado de calidad general. Es posible que aquella deba mucho al taller y que esta sea obra de algún discípulo o seguidor. Para un mejor conocimiento de la obra de este importante pintor filipino es necesario consultar Joaquin, Nick y Santiago, Luciano P. R. : The world of Damián Domingo. Manila, Metropolitan Museum, 1990.

${ }^{17}$ Recientemente se han localizado nuevas pinturas murales en el baptisterio de la misma iglesia de Santa Ana, que han sido seguidamente restauradas. Según el doctor Laya, uno de los máximos conocedores del edificio, existe una clara relación entre la escena del Bautismo de Cristo descubierta y las pinturas del camarín. Un estudio en profundidad de las mismas podrá arrojar luz sobre las que se tratan en este trabajo.
} 
Lo que sí parece claro es el uso de grabados flamencos de comienzos del siglo XVII en la composición. Esta posibilidad no es en absoluto extraña, ya que la generación de grabadores que se desarrolló desde los primeros años del siglo XVIII hasta su segunda mitad conoció estampas y grabados flamencos, italianos y por supuesto españoles que llegaban a Filipinas y en algunos casos eran copiados literalmente. Este corpus gráfico seguiría a disposición de los distintos talleres de la capital a finales del siglo XVIII y son la base de las pinturas que se presentan.

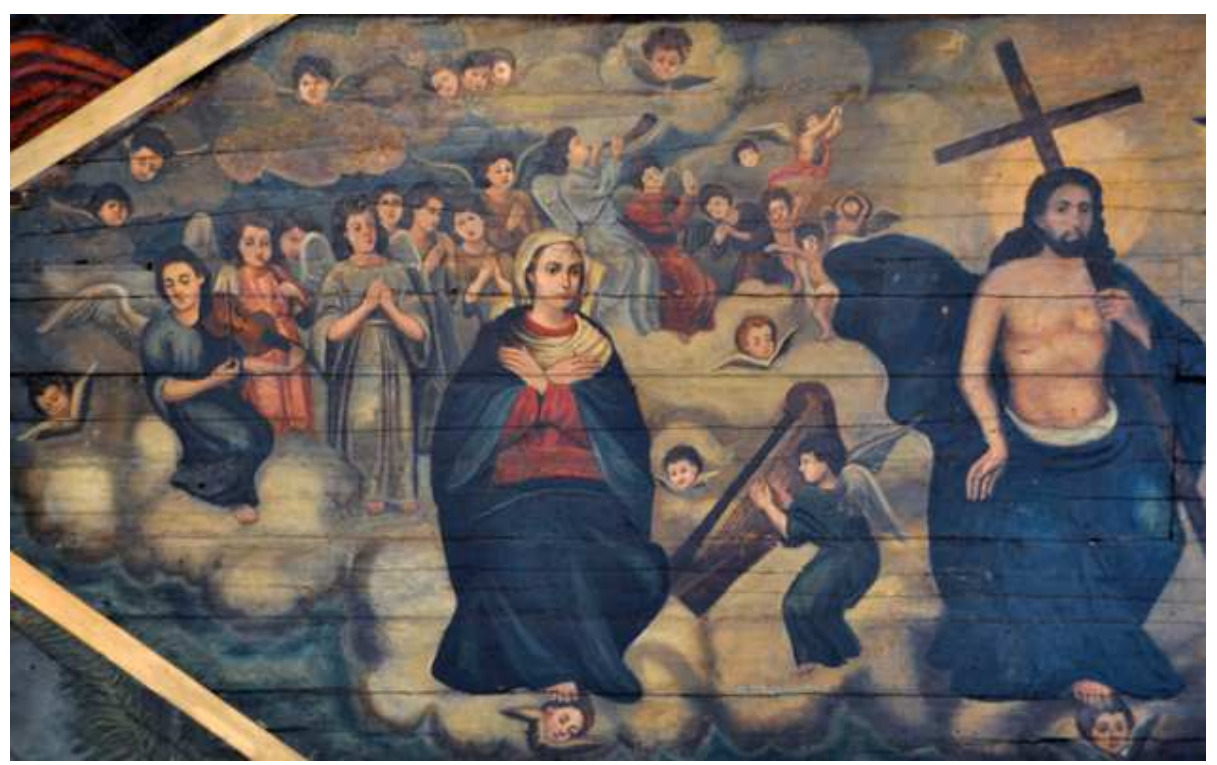

Fig. 1. Camarín de la Virgen de los Desamparados. Santa Ana de Sapa. Manila. Detalle del lado izquierdo de la tabla superior.

Las pinturas pueden dividirse en dos grupos. En primer lugar la portada de acceso al camarín, con San Miguel y San Gabriel arcángeles en los flancos y la adoración del Nombre de María en las hojas de las puertas, todo ello rematado con la corona real. En segundo lugar está el propio techo del camarín, que se forma gracias a nueve tablas. La que cierra la estructura, que será la que se analizará más adelante, es una representación de la Trinidad. El resto de las tablas muestra escenas de la vida de María y alguna de la de Cristo, aunque actualmente no están colocadas de forma correlativa, lo que seguramente se deba a una reubicación poco afortunada. Al presente se suceden de la siguiente manera: Bautismo de Cristo, Cristo entre los doctores, Presentación en el templo, los Reyes Magos -parte de la escena del Pesebre-, la Huída a Egipto, la Adoración de los Pastores, los Desposorios de la Virgen, la Virgen y San José -en el Pesebre que debería ir junto a los Reyes-, la Circuncisión y la Visitación ${ }^{18}$. De todas formas la colocación de las mismas no influye en la interpretación de la iconografía musical del programa. Entre las distintas tablas que forman el techo, sólo la que cierra la estructura presenta representaciones musicales. La obra manileña tiene algunos

\begin{tabular}{|c|c|}
\hline Adoración de los pastores & Desposorios \\
\hline Reyes & Huida a Egipto \\
\hline Reyes & Circuncisión \\
\hline Presentación & Isabel \\
\hline Cristo entre los doctores & Bautismo \\
\hline 123 & Eikon / Imago 1 (2012 / 1) ISSN 2254-8718 \\
\hline
\end{tabular}


paralelos, en lo que a iconografía musical se refiere, en iglesias cercanas que han servido para contextualizarla. Los coros de ángeles más relevantes localizados hasta el momento en las inmediaciones son el conservado en un lienzo del Pesado de almas conservado en la iglesia de Paete, así como la Imposición de los escapularios por la Virgen del Carmen, relieve conservado en la iglesia de Nagcarlan. Observando la complejidad en distintos sentidos de la iconografía de la pieza, se ha visto conveniente trabajar, en primer lugar, los instrumentos por tipos $\mathrm{y}$, en segundo lugar, las agrupaciones entre los mismos y su relación con la organización de la propia obra.

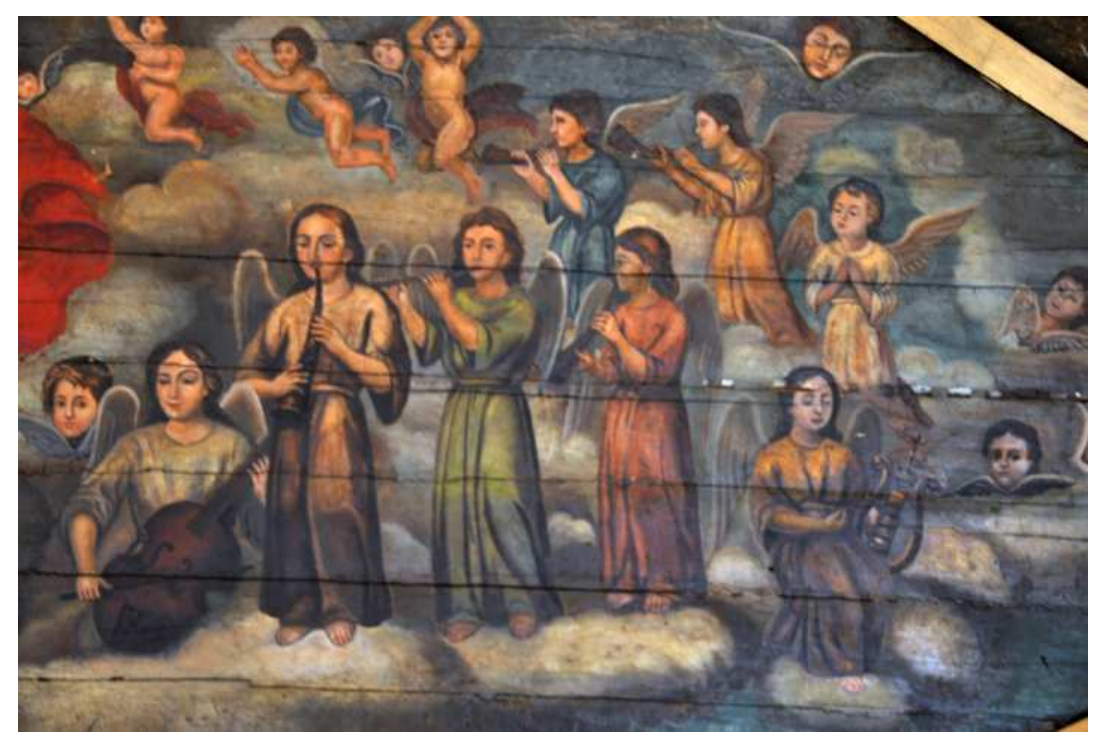

Fig. 2. Camarín de la Virgen de los Desamparados. Santa Ana de Sapa. Manila. Detalle del lado derecho de la tabla superior.

En primer lugar, por número, hay que analizar el grupo de aerófonos. Un total de siete se distribuyen a lo largo del friso creado por los dos conjuntos instrumentales. Esta inclinación por los aerófonos puede relacionarse con el mundo militar, aunque el nulo detallismo y la variedad de instrumentos hace pensar que el artista no tenía una experiencia directa con lo dibujado. Por tanto, avanza la posibilidad de que se inspirara en un grabado que sencillamente simplificara. Los aerófonos de la composición parecen ser bien chirimías bien cornetos. El uso de las primeras en la ciudad de Manila está documentado desde el siglo XVII, utilizándose en grupo para llamar la atención de la ciudadanía para la lectura del pregón. Solía estar acompañado de atabales, que, por otra parte, serían impropios de la composición, y de un clarín "para mayor notoriedad"19.

De todos los instrumentos presentados por el artista, sin duda son las dos arpas las más interesantes, ya no solo por los modelos utilizados, sino por el desarrollo de este instrumento en el archipiélago dentro de la música profana. El arpa se convirtió en una seña de identidad de las mujeres indígenas en las fiestas filipinas. Esto produjo una enorme diversificación de modelos organológicos, entre los cuales destacan el arpa ilocano-cebuana y la bisaya, recientemente estudiadas ${ }^{20}$. El grado de perfeccionamiento que obtuvieron en Filipinas la hace común en los, por otra parte, escasos coros de ángeles músicos conservados.

${ }^{19}$ Alva Rodríguez, Inmaculada. Vida municipal en Manila (siglos XVI-XVII). p. 216.

${ }^{20}$ Grauberger, Stephen L. Diatonic harp of the Philippines: an historical overview and an organological comparison of the Cebuano-bisayan harp. Tesis presentada en la Universidad de Hawaii en 1994. 


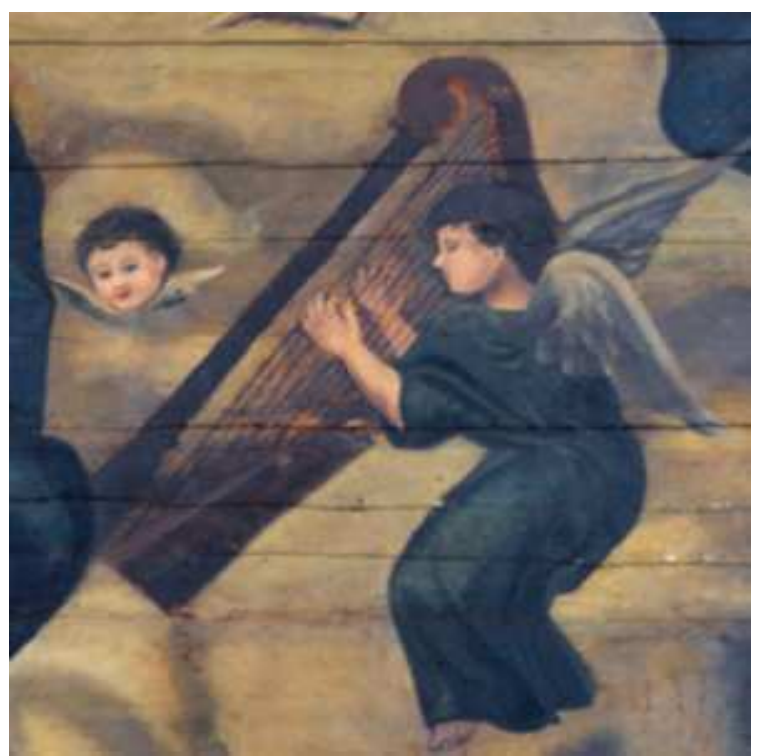

Fig. 3. Camarín de la Virgen de los Desamparados. Santa Ana de Sapa. Manila. Tabla superior. Arpista.

La presencia del arpa en primer plano, separada del conjunto de ángeles y entre la Virgen y Cristo resucitado, debe entenderse como una solución compositiva, más que como una demostración de la raigambre de su interpretación, ya que en otros ejemplos que han podido consultarse el instrumento queda en muchos casos escondido entre la maraña de otros instrumentos, incluso cuando la variedad orquestal y la precisión organológica son mayores. De todas formas, la insuficiente precisión de los artistas hace difícil distinguir en las representaciones las variantes organológicas de las arpas de las distintas provincias, lo que podría ser muy útil para la datación de las piezas ${ }^{21}$. La presente, en caso de tenerla que adscribir a alguna de las variedades estudiadas en las islas, tiene un parecido importante con algunas arpas ilocano-cebuanas aún conservadas. La elección de este tipo regional frente al arpa bisaya quizás pueda encontrarse, primero en su cercanía con la capital, y en segundo lugar, en su mayor homogeneidad tipológica. Un rasgo común entre el modelo ilocano y la que parece representarse en Santa Ana es la decoración de la voluta del puente justo encima de la columna ${ }^{22}$. Sea un modelo organológico u otro, hay rasgos generales que sí son claros en la representación y a la vez muy interesantes. Quizás el punto más reseñable sea el de la inclinación del instrumento con respecto al ángel. La posición es bastante cercana a la que aparece en algunas fotografías de arpistas filipinas de finales del siglo XIX. Por tanto, esta técnica en el archipiélago parece ser al menos tan antigua como la cronología dada para el techo de Santa Ana.

Lo que no es tan común es la aparición, en primer lugar de dos arpas en la misma composición, y en segundo lugar, de una posible cítara o guitarra-lira. El único caso que se ha conseguido recoger es el ya citado de Nagcarlan, donde un ángel porta un arpa en el coro, mientras que el rey David porta su atributo, pero ninguna de ellas es una cítara.

${ }^{21}$ Calvo-Manzano, María Rosa y Martínez Shaw, Carlos. El arpa en el contexto musical de Hispanoamérica y Filipinas a partir de la Era del Descrubrimiento. Madrid: Asociación Arpista Ludovico, 1993. También véase Grauberger, Stephen L. Diatonic harp of the Philippines...

${ }^{22}$ Además de todo esto hay un motivo geográfico claro, y es que si se acepta el título de ilocanocebuano, se estarían agrupando todas las arpas de la isla de Luzón (en cuyo centro se encuentra Manila, y al norte Ilocos), y la isla de Cebú, es decir, la zona más españolizada del archipiélago. Esto tendría además su reflejo lingüístico, ya que en ilocano el arpa se designa con el préstamo castellano (usando arpa o harpa), mientras que en bisaya se utiliza la voz alpa o alfa. 
El tipo de cítara que aparece en la composición tiene cierta relación con la que aparece en el monumento conservado frente a la iglesia de Binondo. Aunque podría haber alguna relación entre la escultura pública y el techo del camarín, parece improbable, siendo más plausible que el pintor se basase en un grabado, mientras que el escultor tomase el modelo de la propia escultura mitológica, que el neoclasicismo español había retomado. Siguiendo con la cítara de Santa Ana, el pintor ha tenido el esmero de pintar el plectro en la mano derecha del intérprete, lo que puede deberse a un seguimiento escrupuloso del grabado, lo cual es lo más probable, o a un conocimiento directo de la práctica musical de este instrumento. Hay un motivo de peso para decantarse por la primera opción y es el escaso nivel de detallismo que tiene en sí el instrumento, ya que no han podido averiguarse el número de cuerdas. Por otra parte no se muestra la caja de resonancia, parte fundamental del instrumento.

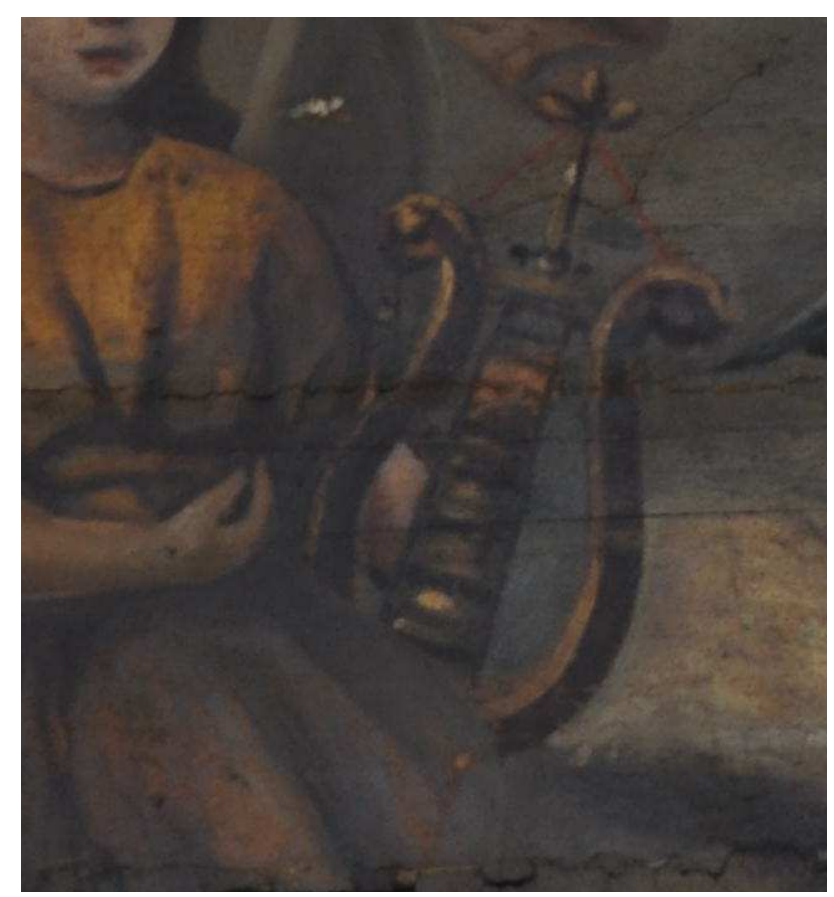

Fig. 4. Camarín de la Virgen de los Desamparados.

Santa Ana de Sapa. Manila. Tabla superior. Detalle de la cítara.

Una segunda posibilidad es que se trate de una guitarra-lira, instrumento de origen francés muy difundido a principios del siglo XIX también en territorio hispano. Esto explicaría el traste que parece quererse representar, no sin notables deficiencias. El enlace con Filipinas sería más probablemente la propia presencia francesa en las Indias Orientales. Los tradicionales contactos culturales entre el archipiélago y ciudades como Pondicherry permitirían aventurar el posible conocimiento de este instrumento en lugares tan remotos.

Debe señalarse que en documentación recientemente localizada se ha podido sustentar la importancia de este tipo de instrumentos en la música litúrgica filipina. En 1782, en el coro de la iglesia de los Santos Reyes del Parián, también en el cinturón de arrabales de Manila, se inventariaron un total de dos arpas, cada una de ellas con una utilidad distinta. Por un lado, la grande, de la que desgraciadamente no tenemos sus dimensiones, y la pequeña, que se utilizaba para el rosario ${ }^{23}$. No hay noticias de que en el resto de templos se conservaran, aun cuando sí aparecen tanto violines, como

${ }^{23}$ AGI, FILIPINAS, 652. 38v. 
órganos. En ninguna de ellas aparecen instrumentos de viento, que debían adscribirse más a la música profana.

Vistos los aerófonos y las arpas, el tercer grupo a analizar es el de la familia de los violines. En este coro aparecen dos, un violín en el coro de la Virgen y un violón en el de Dios Padre. El uso de estos instrumentos en Filipinas, aunque no está muy estudiado, ha podido documentarse recientemente desde fecha muy temprana ${ }^{24}$. Además, actualmente se conserva en el Museo Nacional de Antropología de Madrid un ejemplar de violín realizado en el archipiélago con madera de bambú, mostrando características organológicas propias muy diferentes a los comunes en Europa ${ }^{25}$. De todas formas, los que han podido localizarse representados se atienen a los modelos occidentales, no habiéndose encontrado ninguno que responda a las formas autóctonas. Igualmente parece muy interesante observar el inventario de las iglesias de los arrabales de Manila, realizados durante una visita ya citada ${ }^{26}$. En Binondo se conservaba un violón viejo, mientras que en la iglesia de los Santos Reyes del Parián se inventariaron dos violines y un violón, que habían sido restaurados, debido a su estado. Esto muestra que su existencia en el cordón de arrabales que rodeaban Intramuros era común, aunque quizás su lamentable estado dé noticia de un cambio de prácticas en la música litúrgica filipina ${ }^{27}$. Ya se ha comentado el escaso detallismo del artista, pero parece claro que ambos instrumentos de cuerda frotada parecen vincularse con la familia de los violones.

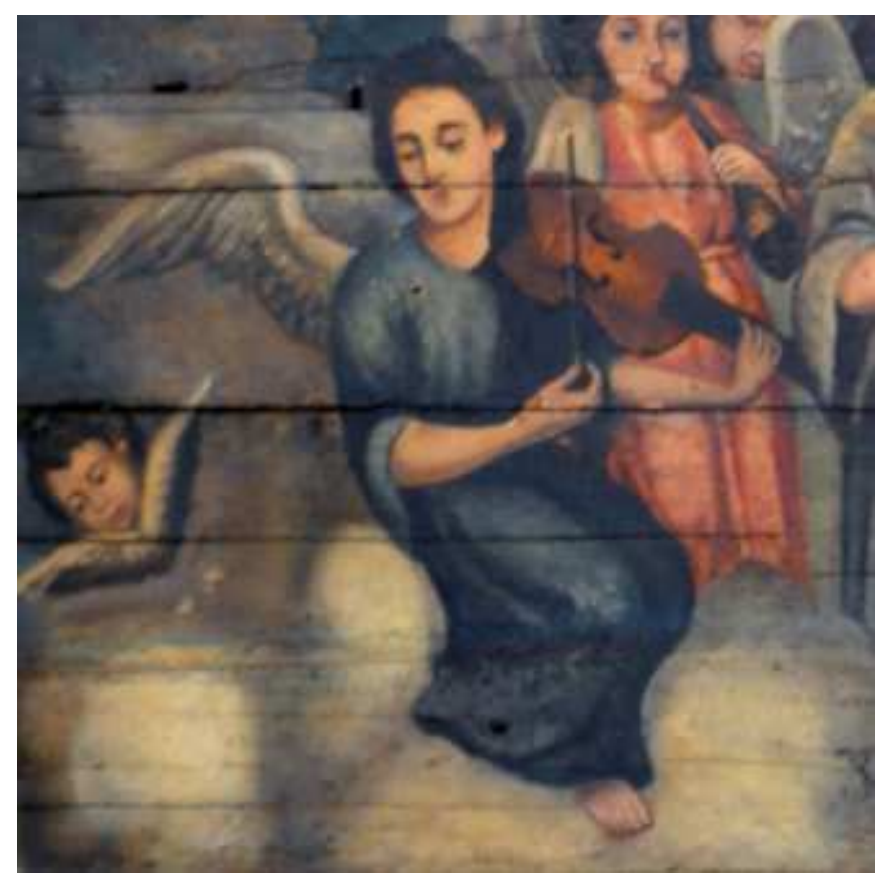

Fig. 5. Camarín de la Virgen de los Desamparados.

Santa Ana de Sapa. Manila. Detalle del violinista.

\footnotetext{
${ }^{24}$ Recientemente se ha podido documentar la existencia de un coro de tagalos y una orquesta de nueve indios negros dirigida por el capitán Esteban Rodríguez de Figueroa para la iglesia jesuítica de Manila. Luengo Gutiérrez, Pedro: Arquitectura conventual en Manila, 1575-1645. Manuscrito inédito. Sevilla, 2008. P. 123.

${ }^{25}$ Violín. Bambú y madera. Tagalog. Centro de Luzón. Museo Nacional de Antropología. Citado por Romero de Tejada, Pilar: "Filipinas, mosaico cultural", España y Filipinas, 1.898. Cádiz, 1998, p. 33.

${ }^{26}$ AGI, FILIPINAS, 652. Además aparecen arpas, que han sido tratadas ya, y algunos órganos, tales como los de Santa Cruz, Santos Reyes del Parián y Binondo.

${ }^{27}$ Para el caso musical de Quiapo debe consultarse Brillantes-Silvestres, Ma Patricia. "Music in The Heart of Manila: Quiapo from the Colonial Period to Comtemporary Times: Tradition, Change, Continuity". 


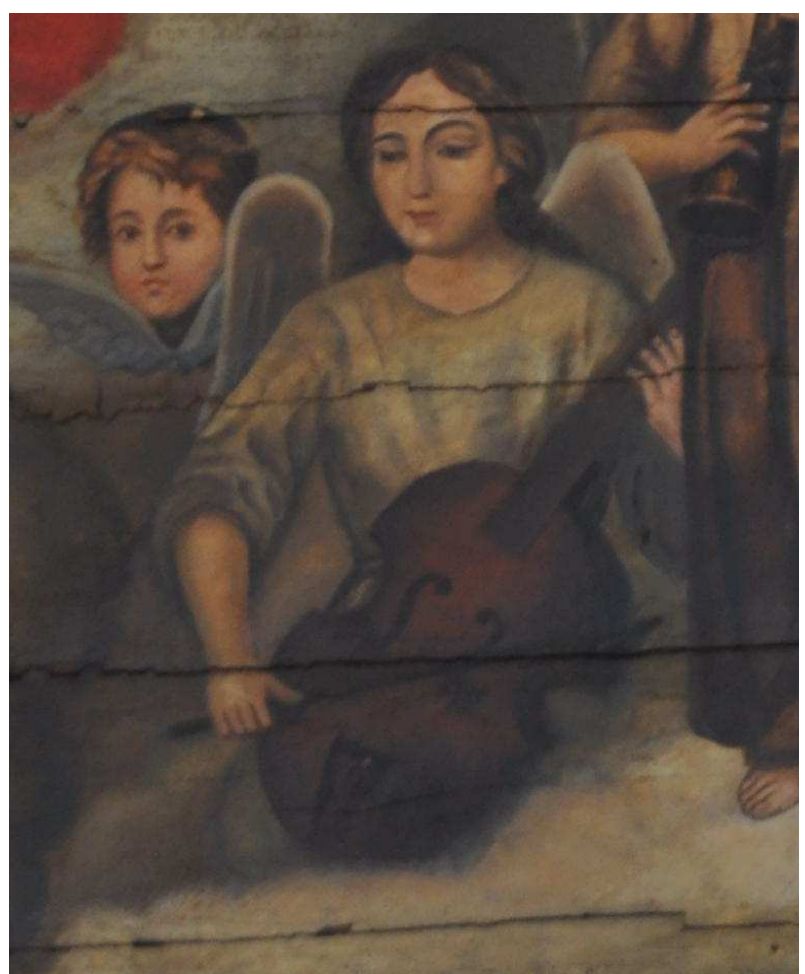

Fig. 6. Camarín de la Virgen de los Desamparados. Santa Ana de Sapa. Manila. Detalle de la viola da gamba.

En casos como estos, en los que se trata de composiciones de cierta complejidad, el análisis de los instrumentos de forma pormenorizada no es suficiente, siendo recomendable ver si el artista está intentando mostrar algún vínculo entre ellos o con algún personaje. La composición pictórica tiene en el centro la representación de la Trinidad, con Dios Padre a la derecha y Dios Hijo a la izquierda. En el coro de la derecha se encuentran en este orden el violón, tres chirimías y la cítara, en primer plano, mientras que algunas cornetas pueden entreverse detrás. El violonchelo podría estar conectado con la figura de Dios Padre, aunque su posición en un extremo de la nube es característica de multitud de coros de ángeles. La agrupación de tres chirimías no es extraña, ya que es la formación tradicional no solo en Filipinas, sino como se ha documentado recientemente, también en la vecina $\mathrm{Macao}^{28}$. La cítara es más difícil de explicar en el contexto que se está exponiendo y teniendo en cuenta que se coloca en primerísimo plano, separado del resto, puede tratarse de un rasgo original por parte del pintor. En el coro de la izquierda, con menor sentido de unidad, ya que los ángeles músicos se encuentran entre las figuras de Cristo y la Virgen, pueden observarse un arpa, un violín y alguna corneta en segundo plano. No parece baladí que rodeen a la Virgen el instrumento característico de la mujer filipina y el soprano de la familia de los violines más clásico de las representaciones marianas.

Una vez analizada la iconografía musical del techo del camarín de Santa Ana de Sapa, será interesante observar algunos aspectos aplicables a futuros estudios sobre el tema en la zona. En primer lugar, resulta evidente que la iconografía musical localizable en Filipinas tiene dos fuentes fácilmente distinguibles en muchos casos. Por un lado se encuentran representaciones de instrumentos cotidianos en Filipinas, lo que suele llevar

${ }^{28}$ Luengo, Pedro: "Fiestas por el recibimiento en Macao de las reliquias del mártir André Cochinchina (1644)”. Huarte de San Juan. Geografía e Historia, 15. Pamplona, 2008, pp. 211-220. 
aparejado una mayor concisión en los detalles y una natural tendencia a los prototipos organológicos autóctonos. Por otro lado, pueden encontrarse traslaciones directas desde grabados europeos antiguos, lo que suele llevar aparejado un escaso nivel de detalle, así como representaciones de instrumentos absolutamente en desuso en el archipiélago, como la cítara, o, en otros casos, el órgano portativo. Por tanto, el estudio de la iconografía musical en el archipiélago permitirá a la vez ahondar en el conocimiento por un lado de aquellos "instrumentos acostumbrados" de cada región, de los que hablan los estatutos franciscanos, y, por otro, de la difusión de grabados europeos en zonas alejadas de la capital. Al parecer, no hubo miedo entre los frailes a representar los más variados instrumentos en los coros de ángeles, llegando incluso a incorporar bandas casi al completo, dejando atrás los modelos antiguos.

En segundo lugar, este techo es demostración de que en general los artistas filipinos no eran demasiado propensos a la representación organológica. Escenas como la Adoración de los Pastores que suelen llevar aparejados distintos instrumentos no los llevan en este caso, y es además ampliable a otros temas como la Tota Pulchra fuera de esta composición. El desarrollo de iconografías musicales suele reducirse, aunque no exclusivamente, a coros celestiales en composiciones de gran tamaño y/o a la representación de Santa Cecilia y el rey David. Si futuras investigaciones confirman este hecho, se produciría un desequilibrio entre la amplia actividad musical filipina y su escasa iconografía musical, lo que se explicaría por la alta dificultad que implica el dibujo de un instrumento de cualquier tipo, y especialmente los más comunes en el archipiélago, el arpa y el órgano. Todo esto unido al escaso desarrollo de la pintura y, en menor medida, de la escultura en madera durante el siglo XVIII.

\section{Fuentes y Bibliografía}

\section{Fuentes}

AGI, FILIPINAS, 652. 38v.

Estatutos y ordenaciones de la Santa Provincia de San Gregorio... Sampaloc: Convento de Nuestra Señora de Loreto, 1753.

\section{Bibliografía}

Alva RodrígueZ, Inmaculada. Vida municipal en Manila (siglos XVI-XVII).

ÁlVAREZ, Rosario. "La iconografía musical latinoamericana en el renacimiento y el barroco: importancia y pautas para su estudio". Interamer, $\mathrm{n}^{\circ}$ 26. Washington, 1993.

Brillantes-Silvestres, Ma Patricia. "Music in The Heart of Manila: Quiapo from the Colonial Period to Comtemporary Times: Tradition, Change, Continuity".

Calvo-Manzano, María Rosa y Martínez Shaw, Carlos. El arpa en el contexto musical de Hispanoamérica y Filipinas a partir de la Era del Descrubrimiento. Madrid: Asociación Arpista Ludovico, 1993.

ESTELla MARCos, Margarita. La escultura barroca de marfil en España: las escuelas europeas y las coloniales. Madrid: CSIC, $1984 .$.

Galende, Pedro G.: Philippine Church Façades. Manila, San Agustín Museum, 2008, p. 15.

Galende, Pedro G. y Trota José, Regalado: San Agustín. Art \& History. 1571-2000”. Hong Kong, 2000. 
GRAUBERGER, Stephen L. Diatonic harp of the Philippines: an historical overview and an organological comparison of the Cebuano-bisayan harp.

HUERTA, Félix de. Estado geográfico, topográfico, estadístico, histórico-religioso... Manila: Sánchez y Cía., 1865.

Irving, David: Colonial Counterpoint: Music in Early Modern Manila. Nueva YorkOxford: Oxford University Press, 2010;

JAVELlanA, René B.: Wood \& Stone for God's greater glory. Jesuit art \& architecture in the Philippines. Quezon City: Ateneo de Manila University Press, 1991;

Joaquin, Nick y Santiago, Luciano P. R. : The world of Damián Domingo. Manila, Metropolitan Museum, 1990.

"Keyboard instruments and instrumentalists in Manila, 1581-1798". Anuario Musical, 60. Madrid, 2005, pp. 27-40.

KUBLER, George. Arquitectura de los siglos XVII y XVIII, Ars Hispaniae XIV, Madrid, 1957;

KuBlER, George. "El camarín del Siglo de Oro" en Cuadernos Hispanoamericanos, $\mathrm{n}^{\circ}$ 140-141. 1961, pp. 239-245.

LuENGO, Pedro: "Fiestas por el recibimiento en Macao de las reliquias del mártir André Cochinchina (1644)". Huarte de San Juan. Geografía e Historia, 15. Pamplona, 2008, pp. 211-220.

LuENGO, Pedro. "Noticias sobre obras en la iglesia de Tondo en el siglo XVIII". Laboratorio de Arte. Sevilla, 2010.

LUENGO GUTIÉRREZ, Pedro: Arquitectura conventual en Manila, 1575-1645. Manuscrito inédito. Sevilla, 2008, p. 123.

Martínez Shaw, Carlos y Alfonso Mola, Marina (dir.): Catálogo de la exposición El Galeón de Manila. Sevilla: MECD y Focus-Abengoa, 2000.

Morales, Alfredo J. (dir.): Catálogo de la exposición Filipinas, puerta de Oriente. De Legazpi a Malaspina. Madrid: SEACEX, 2003. Ruiz Gutiérrez, Ana. El tráfico artístico entre España y Filipinas (1565-1815). Granada: Universidad de Granada, 2005.

Moreno Garbayo, Justa. Fiestas en Manila año 1825. Madrid: Patrimonio nacional, 1977.

REYES, Octavio. Iconografía musical novohispana en la región central de Puebla. Puebla: Universidad Iberoamericana, Plantel Golfo-Centro, 1995;

ROMERO DE TEJADA, Pilar: "Filipinas, mosaico cultural", España y Filipinas, 1.898. Cádiz, 1998, p. 33.

RouBINA, Eugenia. "Fuentes iconográficas en el estudio de las prácticas instrumentales en la música popular novohispana del siglo XVIII". Cátedra de Artes, n 4. Santiago de Chile: Pontificia Universidad Católica de Chile, 2007.

"Some Notes on Viols in the Philippines". Chelys Australis: Australian Viola da Gamba Society Journal, 4 (2004). Pp. 13-16;

Trota José, Regalado: Simbahn: Church art in colonial Philippines. 1565-1898. Manila: Ayala Foundation, 1992.

VV.AA.: Manila 1571-1898. Occidente en Oriente. Madrid: Ministerio de Fomento, 1998

VV. AA.: Igkas Arte. The Philippine Arts during the Spanish Period. Manila: CCPAECI, 1998.

VV. AA.: Santa Ana Church. A Historical Guide. Manila: Cofradía de la Inmaculada Concepción Foundation, 2008. 